\title{
Effect of early palliative care on quality of life in patients with non-small-cell lung cancer
}

\author{
H. Zhuang $\mathrm{MB}^{*}{ }^{*}$ Y. Ma $\mathrm{MB}_{1}^{+}$L. Wang $\mathrm{MB}^{\neq}$and H. Zhang $\mathrm{MB}^{\S}$
}

\begin{abstract}
Background Patients with metastatic non-small-cell lung cancer (NSCLC) experience great pain and stress. Our study aimed to explore the effect of early palliative care on quality of life in patients with NSCLC.

Methods A total of 150 patients were randomly divided into two groups: control group with conventional care and study group with early palliative care. The quality of life (QOL) rating scale and self-rating scale of life quality (SSLQ) were used to analyze the patients' quality of life. The Hospital Anxiety and Depression Scale-D/A (HADS-D/A) and Patient Health Questionnaire 9 (PHQ-9) were used to analyze the patients' mood. Pulmonary function indexes of peak expiratory flow (PEF), functional residual capacity (FRC), and trachea-esophageal fistula $25 \%$ (TEF $25 \%$ ) were analyzed using the lung function detector.
\end{abstract}

Results The QOL and SSLQ scales scores of patients receiving early palliative care were significantly higher than those in the control group $(p<0.05)$. Moreover, the questionnaire results of the HADS-D/A and PHQ- 9 were better in patients receiving palliative care than in the control group $(p<0.05$ or $p<0.01)$. In addition, analytical results of pulmonary function showed that the levels of PEF, FRC, and TEF $25 \%$ in patients assigned to early palliative care were remarkably higher than those in the control group $(p<0.01$ or $p<0.001)$.

Conclusions These data demonstrate that early palliative care improves life quality, mood, and pulmonary function of NSCLC patients, indicating that early palliative care could be used as a clinically meaningful and feasible care model for patients with metastatic NsCLC.

Key Words Palliative care, non-small-cell lung cancer, HADS-D/A, PHQ-9, pulmonary function

Curr Oncol. 2018 Feb;25(1):e54-e58

www.current-oncology.com

\section{INTRODUCTION}

Unhealthy lifestyles and environmental pollution are producing an increase in the incidence and mortality of non-small-cell lung cancer (NSCLC) year by year ${ }^{1}$. Nonsmall-cell lung cancer, which accounts for over $80 \%$ of lung cancer, has high morbidity and mortality all around the world ${ }^{2}$. It is the kind of debilitating disease that may lead to poor quality of life. It has been reported that cancer pain is usually accompanied by anxiety or depression ${ }^{3}$. Numerous studies suggest that the global burden of lung cancer is constantly increasing due to the rise in cancercausing behaviours, and many suffer from great pain, for which there is little remedy. Increasing evidence suggests that some optimal health services are urgently needed in this stage ${ }^{4,5}$.
Palliative care, long-term care that improves the quality of a patient's life and reduces the use of medical services ${ }^{7}$, has been put into use worldwide, focusing on terminal cancer care ${ }^{8,9}$. Palliative care aims to improve patients' symptoms, including family satisfaction with care ${ }^{10}$, receiving assistance with decision making ${ }^{11}$, promoting patients' subjective well-being, and communicating with healthcare providers ${ }^{12}$. Because it needs to meet a variety of needs, palliative care must be comprehensive, multidisciplinary, and patient- or familycentred. However, palliative care is usually delivered to patients late in the course of a disease, when its nursing effect is weakened ${ }^{13}$. At the same time, the misconception that palliative care is "giving up" on life impedes the treatment of cancer when patients are suffering from a terminal illness ${ }^{14}$. 
Currently, several studies have found that late application of palliative care cannot fully improve patients' quality of life ${ }^{15,16}$. It is therefore necessary to provide earlier palliative care services in the course of cancer. In terms of NSCLC, accumulating evidence indicates that palliative care for patients with metastatic NscLc leads to improvements in quality of life, mood, end-of-life care, and possibly survival ${ }^{17}$. Adnan et al. showed that palliative care significantly improved all general aspects of quality of life in patients with lung cancer ${ }^{18}$. Based on these previous studies, we further explored the effect of early palliative care on quality of life in patients with NScLC. Our findings demonstrate that early palliative care can effectively improve life quality, mood, and pulmonary function in patients with NSCLC. These data will provide a new insight for the clinical treatment of NSCLC.

\section{METHODS}

\section{Study Design}

A total of 150 newly-diagnosed NSCLC patients were selected between 20 February 2010 and 20 May 2014 at the First People's Hospital of Xianyang City. Patients diagnosed within the previous eight weeks were eligible to be enrolled and were randomly divided into two groups in a 1:1 radio: one group was treated with early palliative care combined with standardized tumour management, and the control group was treated only with conventional tumour management. The participants in the early palliative care combined with standardized tumour management group were cared for by board-certified palliative care physicians and advanced-practice nurses and were followed once per month until death. The general rules of palliative management were adapted from the National Consensus Project for Quality Palliative Care $^{19}$. The palliative care clinicians used an electronic medical record template to assess patients' physical and psychosocial condition, establish management objectives, and provide corresponding medical services. The study was approved by the First People's Hospital of Xianyang City, and all participants provided written informed consent and accepted standardized tumour management.

\section{Study Patients}

The patients who participated in this study were selected from the outpatient thoracic oncology clinic of the First People's Hospital of Xianyang City. Inclusion criteria were as follows: (i) patients were diagnosed as NSCLC and enrolled in the study within eight weeks; (ii) patients' Eastern Cooperative Oncology Group (ECOG) behaviour status ranged from 0 to 2 ( 0 , asymptomatic; 1 , symptomatic but fully ambulatory; 2 , symptomatic and in bed $<50 \%$ of the day); (iii) patients were able to read and respond to questions. Patients who had previously received palliative care services were not eligible to participate in the study.

\section{Patient Reported Measures}

To assess patients' quality of life in a variety of ways, the Quality of Life (QOL) rating scale and Self-rating Scale of Life Quality (SSLQ) were used to evaluate patients' physical, psychological, social role, responsibility, health self-assessment, and economic situations. Patients' psychological assessment was determined using the Hospital Anxiety and Depression Scale (HADS) and the Patient Health Questionnaire 9 (PHQ-9). The HADs consists of two subscales and includes 14 items. It was used to assess patients' anxiety and depression the week before they participated in the study. The subscale scores range from 0 (no distress) to 21 (maximum distress). Scores $>7$ are considered to be clinically significant. The PHQ-9 was used to estimate depressive syndrome according to the Diagnostic and Statistical Manual of Mental Disorders and includes nine items. Patients who agreed with five items on the PHQ-9 were considered to be depressed. Symptoms needed to be present more than half the time except for the symptom of suicidal thoughts. In addition, pulmonary function indices were analyzed with a lung function detector (CORTEX, Germany), including peak expiratory flow (PEF), functional residual capacity (FRC), and tracheaesophageal fistula 25\% (TEF 25\%).

\section{Data Collection}

All participants completed the basic questionnaire before they were randomly assigned. Twelve weeks after the questionnaires were completed, quality of life, mental state, and pulmonary function of all participants were assessed. If participants were not scheduled for clinic visits, they received the questionnaires in the mail during this period. If participants did not complete the questionnaires, the researchers documented the reasons for this.

\section{Statistical Analyses}

Statistics were analyzed using version 17.0 of SPSS software (SPSS, Inc., Chicago, IL, U.S.A.). Mean, standard deviation (SD), and range were estimated using descriptive statistics. Differences between the early palliative care group and the control group were examined using bilateral Fisher's exact and Chi-squared tests. Continuous variables were analyzed with student's $t$-test. The influences of early palliative care on participants' quality of life were evaluated by multivariate linear regression analyses.

\section{RESULTS}

\section{Baseline Characteristics of Patients}

Of the 210 eligible patients screened to the outpatient thoracic oncology clinic from 20 February 2010 to 20 May 2014, 150 (71.4\%) were enrolled in the study. The enrolled patients were randomly divided into two groups: the control group received conventional tumour management and the study group received early palliative care combined with standardized tumour management. Statistical analysis was used to compare sex, age, education, marital status, and patients' degree of comprehension of the illness between the groups. The results showed the baseline characteristics were well matched and there was no significant difference between the control group and the study group (Table I).

\section{Palliative Care Improved Patient Quality of Life}

The QOL rating scale and SsLQ were used to analyze the influence of palliative care on patient quality of life. The results (Figure 1) indicate that QOL and SSLQ scores were 
TABLE I Comparison of demographic variables of control and study groups

\begin{tabular}{|c|c|c|c|c|}
\hline Group & Variable & Control $(n=78)$ & Study $(n=72)$ & $P$ value \\
\hline \multirow[t]{2}{*}{ Sex } & Male & $72.6 \%$ & $76.1 \%$ & 0.78 \\
\hline & Female & $27.4 \%$ & $23.9 \%$ & \\
\hline Mean age & & 62.4 & 69.2 & 0.46 \\
\hline \multirow[t]{3}{*}{ Education } & Primary & $48.1 \%$ & $47.7 \%$ & \\
\hline & High school & $37.2 \%$ & $35.6 \%$ & \\
\hline & University & $11.1 \%$ & $12.5 \%$ & \\
\hline \multirow[t]{4}{*}{ Marital status } & Single & $10.9 \%$ & $9.1 \%$ & 0.58 \\
\hline & Married & $66.8 \%$ & $70.2 \%$ & \\
\hline & Divorced & $10.2 \%$ & $9.6 \%$ & \\
\hline & Widowed & $10.1 \%$ & $11.1 \%$ & \\
\hline \multirow[t]{3}{*}{ Comprehension degree on illness } & Know nothing about illness and prognosis & $30.4 \%$ & $29.2 \%$ & 0.91 \\
\hline & Understand diagnosis and not understand prognosis & $34.5 \%$ & $33.6 \%$ & \\
\hline & Fully understand diagnosis and prognosis & $35.1 \%$ & $37.2 \%$ & \\
\hline
\end{tabular}

A

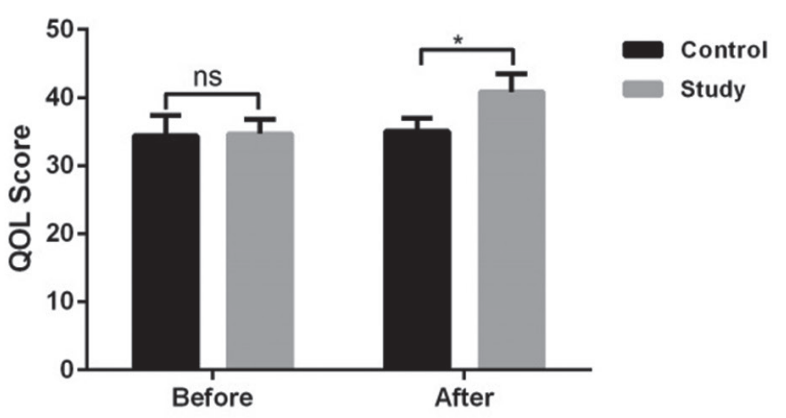

B

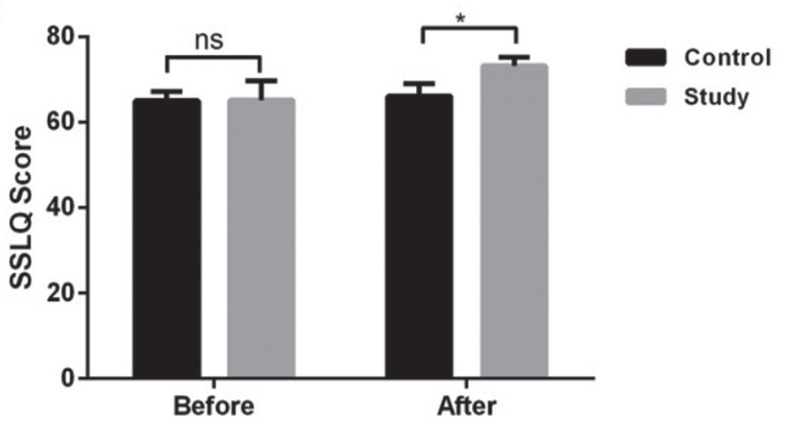

FIGURE 1 Effects of early palliative care on patients' quality of life. One hundred fifty enrolled patients were randomly divided into two groups: the control group received conventional tumour management, and the study group received early palliative care combined with standardized tumour management. Quality of life of NSCLC patients was analyzed using (A) the QOL rating scale and (B) the SSLQ rating scale. Higher scores indicate better quality of life. NSCLC = non-small-cell lung cancer; $\mathrm{QOL}=$ quality of life; SSLQ = self-rating scale of life quality; ${ }^{*} p<0.05$.

significantly higher in the patients assigned to early palliative care than in those in the control group $(p<0.05)$. Taken together, these results suggest that early palliative care could improve the quality of life of NSCLC patients.

\section{Effect of Palliative Care on Patients' Mood}

The percentages of patients with depression at 12 weeks, HADS-D, HADS-A, and PHQ-9, were used to analyze the mood of NSCLC patients. As shown in Figure 2, the percentages of HADS-D, HADS-A, and PHQ-9 were significantly lower ( $p$ $<0.05$ or $p<0.01$ ) in the study group (with palliative care) than in the control group ( $18.8 \%$ vs. $31.6 \%, 16.7 \%$ vs. $27.3 \%$, and $8.9 \%$ vs $15.6 \%$, respectively). These data indicate that palliative care could alleviate the negative emotions of NSCLC patients.

\section{Effects of Palliative Care on Patients' Pulmonary Function}

The pulmonary function indices, PEF, FRC, and TEF $25 \%$, were measured with the lung function detector. The results show that PEF, FRC, and TEF $25 \%$ were significantly lower in the control group than in the healthy group, indicating severely impaired pulmonary function in lung cancer patients, but all three measures were significantly better in the study group (with palliative care) than in the control group ( $p<0.01$ or $p<0.001$, Figure 3 ). Overall, these data indicate that palliative care has the potential to improve pulmonary function in NSCLC patients.

\section{DISCUSSION}

Non-small-cell lung cancer is one of the most common lung cancers, accounting for more than $80 \%$ of all cases worldwide, with a high morbidity and mortality ${ }^{20}$. The majority of NSCLC patients are diagnosed at an advanced stage, and they frequently suffer from physical and emotional distress that seriously impacts their quality of life ${ }^{21}$. Previous studies have shown that the majority of patients with NSCLC have low quality of life, negative emotions, and short survival ${ }^{22}$. Each year, more than 100,000 patients die from NSCLC, and a large number of patients and families suffer from the emotional, physical, and social impacts of the terminal diagnosis ${ }^{23-25}$. Given this situation, appropriate measures such as palliative 
A

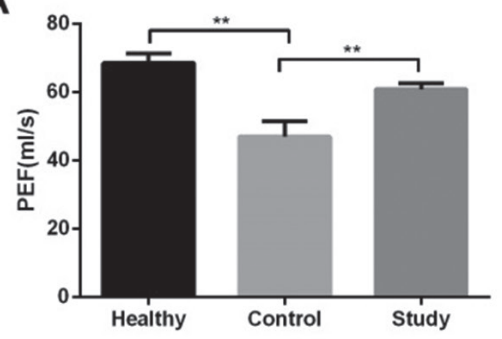

B

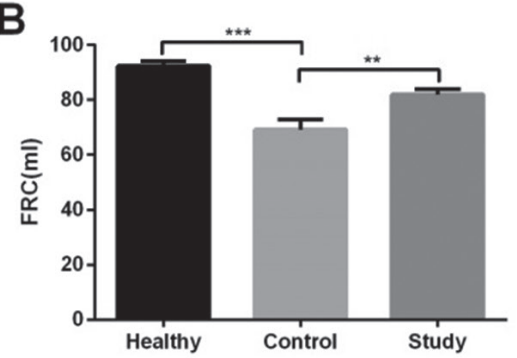

C

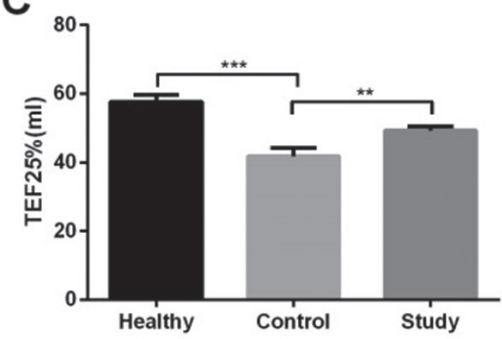

FIGURE 2 Effects of palliative care on the mood of patients. One hundred fifty enrolled patients were randomly divided into two groups: the control group received conventional tumour management, and the study group received early palliative care combined with standardized tumour management. (A) HADS-D (B) HADS-A and (C) PHQ-9 were used to analyze the mood of NSCLC patients.

HADS-D = hospital anxiety and depression scale-D (depression); HADS-A = hospital anxiety and depression scale-A (anxiety); PHQ-9 = patient health questionnaire $9 ;{ }^{*} p<0.05 ;{ }^{* *} p<0.01$.

A

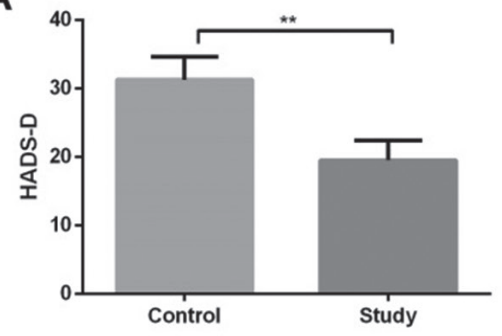

B

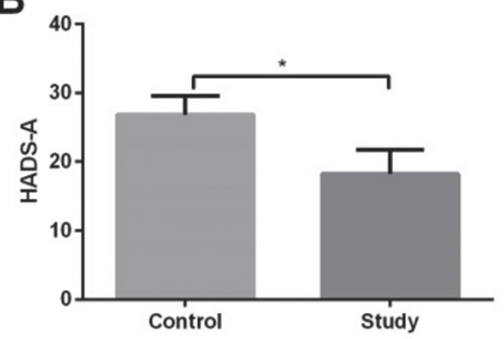

C

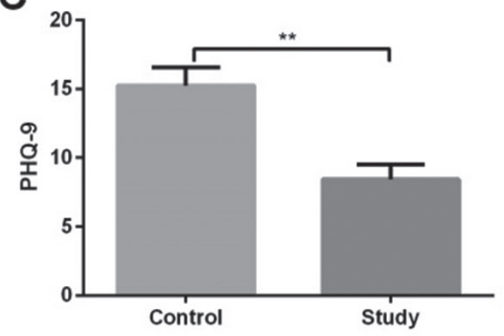

FIGURE 3 Effects of palliative care on pulmonary function of patients. One hundred fifty enrolled patients were randomly divided into two groups: the control group received conventional tumour management, and the study group received early palliative care combined with standardized tumour management. The three pulmonary function indices, (A) PEF, (B) FRC, and (C) TEF 25\%, were measured with a lung function detector. $\mathrm{PEF}=$ peak expiratory flow; FRC = functional residual capacity; TEF $25 \%=$ trachea-esophageal fistula $25 \% ;{ }^{* *} p<0.01 ;{ }^{* * *} p<0.001$.

care are urgently needed to improve the quality of life of these patients.

A recent study has confirmed that palliative care as a supportive care approach could prevent and alleviate pain by means of early identification, positive evaluation, and pain control, thereby improving quality of life of patients and families ${ }^{26}$. The American Society of Clinical Oncology (Asco) declared that early palliative care is becoming important for the treatment of advanced cancers and has also been widely validated as an effective and feasible method for improving quality of life or extending survival time ${ }^{27-29}$. As Temel et al. demonstrated, early palliative care could lead to better management of symptoms, including prolonged survival and stabilization of patients' condition $^{30}$. In consideration of the progressive character of NSCLC, improving quality of life and mood in patients with this illness is a formidable challenge ${ }^{31}$.To date, several studies have been reported that early palliative care for patients with advanced NscLC could improve quality of life, decrease aggressive care at the end of life and possibly enhance survival ${ }^{32,33}$. Early palliative care was also reported to result in less aggressive end-of-life care, including decreased chemotherapy and longer hospice care ${ }^{34}$. Similarly to these previous studies, our results showed significant improvement of quality of life and mood in patients with early palliative care compared with those receiving normal care. At the same time, our study demonstrated that the intervention of early palliative care significantly improved pulmonary function such as PEF, FRC, and TEF 25\%. During our experimental study, more patients intended to participate in the early palliative care group than in the normal group, indicating patients' expectations of good quality life at the end of their lives.

\section{CONCLUSIONS}

Our results showed that patients receiving early palliative care had significantly improved quality of life, mood, and pulmonary function compared with those receiving normal care. The results indicate that early palliative care could be used as a clinically meaningful and feasible care model for patients with metastatic NSCLC.

\section{CONFLICT OF INTEREST DISCLOSURES}

We have read and understood Current Oncology's policy on conflicts of interest disclosure and declare that we have none.

\section{AUTHOR AFFILIATIONS}

*Department of Respiratory Medicine, Weifang People's Hospital, Weifang 261041, China; 'Department of Nursing, The First People's Hospital of Xianyang City, Xianyang 712000, China; ${ }^{\ddagger}$ Department of Oncology, Binzhou City Central Hospital, Binzhou 251700, China; ${ }^{\S}$ Department of Respiratory Medicine, Baoji Central Hospital, Baoji 721008, China. 


\section{REFERENCES}

1. Eguchi T, Bains S, Lee MC, et al. Impact of increasing age on cause-specific mortality and morbidity in patients with Stage I non-small-cell lung cancer: a competing risks analysis. J Clin Oncol 2017;35:281.

2. Wan JW, Gao MZ, Hu RJ, et al. A preliminary study on the relationship between circulating tumor cells count and clinical features in patients with non-small cell lung cancer. Ann Trad Med 2015;3:352.

3. Zhang R, Zhang Y, Wen F, Wu K, Zhao S. Analysis of pathological types and clinical epidemiology of 6,058 patients with lung cancer. Chin J Lung Cancer 2016;19:129-35.

4. Urman A, Hosgood HD. Curbing the burden of lung cancer. Front Med 2016;10:228-32.

5. Rittmeyer A. Quality of life in patients with NSCLC receiving maintenance therapy. Cancers 2015;7:950-62.

6. Kapo JM, Akgün KM. Integrating palliative care into the care of patients with advanced lung cancer. Cancer J 2015;21:434.

7. Albizurivera A, Portman DG, Thirlwell S, Codada SN, Donovan KA. Implementation of NCCN palliative care guidelines by member institutions. Supp Care Cancer 2016;24:929-32.

8. Daveson BA, de Wolf-Linder S, Witt J, et al. Results of a transparent expert consultation on patient and public involvement in palliative care research. Palliat Med 2015;29:939-49.

9. Rak A, Raina R, Suh TT, et al. Palliative care for patients with end-stage renal disease: approach to treatment that aims to improve quality of life and relieve suffering for patients (and families) with chronic illnesses. Clin Kidney J 2017;10:68-73.

10. Kataoka-Yahiro MR, Mcfarlane S, Kealoha M, SyA. Asian and native Hawaiian family caregiver satisfaction with palliative care services in nursing homes. International J Palliat Nurs 2016;22:141.

11. Chand P, Gabriel T, Wallace CL, Nelson CM. Inpatient palliative care consultation: describing patient satisfaction. Permanente J 2013;17:53-5.

12. Lee EM. Palliative Care. Springer International Publishing; 2016.

13. Salins N, Patra L, Rani MU, et al. Integration of early specialist palliative care in cancer care: survey of oncologists, oncology nurses, and patients. Ind J Palliat Care 2016;22:258-65.

14. Beernaert K, Deliens L, Vleminck A, et al. Is there a need for early palliative care in patients with life-limiting illnesses? Interview study with patients about experienced care needs from diagnosis onward. Am J Hosp Palliat Care 2016;33:489-97.

15. Bilaçero lu S. Role of palliative care in improving the quality of life in elderly with advanced lung disease. Curr Geriatr Rep 2016;5:103-9.

16. Nowicki A, Farbicka P, Krajnik M. Dejection and selfassessment of quality of life in patients with lung cancer subjected to palliative care. Contemp Oncol 2016;19:491-5.

17. Temel JS, Greer JA, Muzikansky A, et al. Early palliative care for patients with metastatic non-small-cell lung cancer. $N$ Engl J Med 2010;363:733-42.

18. Delibegovic A, Sinanovic O. The influence of pallative care on the level of anxiety and depression in lung cancer patients. Med Arch 2013; 67:263-65.
19. Colby WH, Dahlin C, Lantos J, Carney J, Christopher M. The National Consensus Project for Quality Palliative Care Clinical Practice Guidelines Domain 8: ethical and legal aspects of care. HEC Forum 2010;22:117-31.

20. Jenssen C, Annema JT, Clementsen P, Cui XW, Borst MM, Dietrich CF. Ultrasound techniques in the evaluation of the mediastinum, part 2: mediastinal lymph node anatomy and diagnostic reach of ultrasound techniques, clinical work up of neoplastic and inflammatory mediastinal lymphadenopathy using ultrasound techniques and how to learn mediastinal endosonography. J Thorac Dis 2015;7:E439.

21. Hirsh V. Is the evaluation of quality of life in NscLC trials important? Are the results to be trusted? Front Oncol 2014;4:173.

22. Movsas B, Moughan J, Sarna L, et al. Quality of life supersedes the classic prognosticators for long-term survival in locally advanced non-small-cell lung cancer: an analysis of RTOG 9801. J Clin Oncol 2009;27:5816-22.

23. Torre LA, Bray F, Siegel RL, Ferlay J, Lortet-Tieulent J, Jemal A. Global cancer statistics, 2012. CA Cancer JClin 2015;65:87-108.

24. Chen W, Zheng R, Baade PD, et al. Cancer statistics in China, 2015. CA Cancer J Clin 2016;66:115-32.

25. Siegel RL, Miller KD, Jemal A. Cancer statistics, 2015. CA Cancer J Clin 2015;65:5-29.

26. Sharma N, Sharma AM, Wojtowycz MA, Wang D, Gajra A. Utilization of palliative care and acute care services in older adults with advanced cancer. J Geriatr Oncol 2016;7:39-46.

27. Bakitas M, Lyons KD, Hegel MT, et al. Effects of a palliative care intervention on clinical outcomes in patients with advanced cancer: the Project ENABLE II randomized controlled trial. JAMA 2009;302:741-9.

28. Irwin KE, Greer JA, Khatib J, Temel JS, Pirl WF. Early palliative care and metastatic non-small cell lung cancer: potential mechanisms of prolonged survival. Chron Resp Dis 2013;10:35-47.

29. Temel JS, Greer JA, Muzikansky A, et al. Early palliative care for patients with metastatic non-small-cell lung cancer. New Engl J Med 2010;363:733-42.

30. Temel JS, Greer JA, Admane S, et al. Longitudinal perceptions of prognosis and goals of therapy in patients with metastatic non-small-cell lung cancer: results of a randomized study of early palliative care. J Clin Oncol 2011;29:2319-26.

31. Gross M, Kozak D, Laufer BZ, Weiss EI. Manual closing torque in five implant abutment systems: an in vitro comparative study. J Prosth Dent 1999;81:574-8.

32. Parekh HD, Tullio K, Elson P, et al. The effect of routine early palliative care (PC) consultation on aggressiveness of care at the end of life (EOL) in patients with advanced non-small cell lung cancer (NSCLC). J Clin Oncol 2016;34 [Epub ahead of print].

33. Philip J, Hudson P, Bostanci A, et al. Metastatic non-small cell lung cancer: a benchmark for quality end-of-life cancer care? Med J Aust 2015;202:139-43.

34. Tsai CJ, Li J, Gonzalez-Angulo AM, et al. Outcomes after multidisciplinary treatment of inflammatory breast cancer in the era of neoadjuvant HER2-directed therapy. Am J Clin Onco 2015;38:242. 